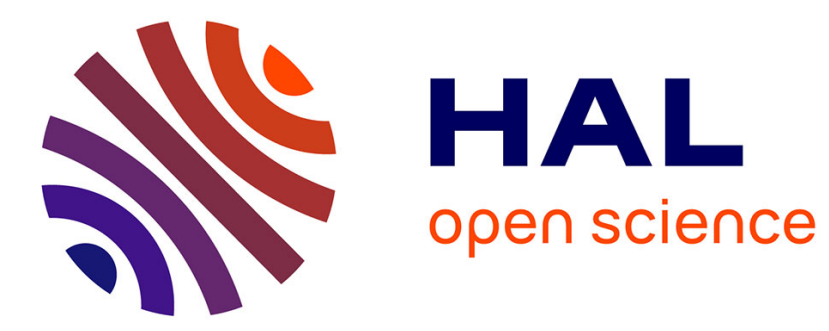

\title{
Distance and angular resolutions improvement for a ground-based radar imager
}

\author{
R. Rouveure, P. Faure, Marion Jaud, M.O. Monod, L. Moiroux-Arvis
}

\section{To cite this version:}

R. Rouveure, P. Faure, Marion Jaud, M.O. Monod, L. Moiroux-Arvis. Distance and angular resolutions improvement for a ground-based radar imager. IEEE International Radar Conference (RADAR), Oct 2014, Lille, France. 6 p. hal-01122332

\section{HAL Id: hal-01122332 \\ https://hal.science/hal-01122332}

Submitted on 3 Mar 2015

HAL is a multi-disciplinary open access archive for the deposit and dissemination of scientific research documents, whether they are published or not. The documents may come from teaching and research institutions in France or abroad, or from public or private research centers.
L'archive ouverte pluridisciplinaire HAL, est destinée au dépôt et à la diffusion de documents scientifiques de niveau recherche, publiés ou non, émanant des établissements d'enseignement et de recherche français ou étrangers, des laboratoires publics ou privés. 


\title{
Distance and angular resolutions improvement for a ground-based radar imager
}

\author{
R. Rouveure, P. Faure, M. Jaud, M-O. Monod, L. Moiroux-Arvis \\ UR TSCF \\ Irstea \\ Aubière, France \\ raphael.rouveure@irstea.fr
}

\begin{abstract}
PELICAN is a ground-based radar imager developed for outdoor perception in the domain of mobile robotics and environmental monitoring. In order to obtain radar images with higher spatial resolution, the basic FFT tool for spectral analysis has been replaced and completed: (i) high resolution spectrum analysis with ESPRIT parametric method for distance resolution; (ii) Richardson-Lucy deconvolution to take into account the convolution introduced by the antenna beam width and to improve the angular resolution. Results obtained with simulated data and real data provided by the PELICAN radar are presented and discussed.
\end{abstract}

Keywords-FMCW radar; distance resolution; angular resolution; ESPRIT algorithm; Richardson-Lucy deconvolution

\section{INTRODUCTION}

For many applications such as mobile robotics or environmental management, perception remains a critical point when operating in a natural environment. Laser and video based systems are common optical based sensors, particularly well adapted in indoor environments. However, these systems are affected by visibility conditions that are often poor in outdoor environments: day/night cycles change illumination, weather phenomena as fog, rain or dust may deteriorate the quality of information provided [1,2]. Considering these operating conditions, radar provides an alternative solution because data can be acquired independently of atmospheric conditions and time of the day.

We developed a small-sized radar imager easy to embark on board of different ground vehicles. The potentialities of this radar, called PELICAN, have been evaluated through mobile robotics [3] and environmental management applications [4]. The distance and angular resolutions of the radar are limited due to currently adopted radar configurations (dimensions of the antenna, bandwidth of the transmitted signal) and signal processing methods (FFT spectral analysis). The objective of the paper is to present solutions in order to improve both resolutions.

The paper is organized as follows. In section II, we describe the PELICAN radar imager, which is based on a FMCW radar and an associated mapping algorithm. In section III and IV, the proposed solutions to increase distance and angular resolutions are presented: high resolution frequency analysis based on the
ESPRIT algorithm for distance resolution; Richardson-Lucy algorithm for angular resolution. Results obtained with PELICAN radar data are shown in the last section.

\section{PELICAN RADAR IMAGeR}

The developed ground-based mobile mapping system is based on two main elements: (i) the PELICAN microwave radar, and (ii) the R-SLAM mapping algorithm.

The PELICAN radar is a Frequency Modulated Continuous Wave -FMCW- radar, which is well indented for short and medium range applications $[5,6]$. The principle of FMCW radar consists to mix the emitted signal following a linear modulation waveform, with the received signal delayed after reflection on the targets present within the view of the antenna. The expression of the beat signal $S_{b}$ produced by the radar is of the form [7]:

$$
S_{b}(t)=k \sum_{i} V_{e} V_{r i} \cos \left\{2 \pi\left(\begin{array}{c}
2 / c\left(\Delta F F_{m} R_{i}+f_{0} V_{i}\right) t \\
+2 / c \Delta F F_{m} V_{i} t^{2}+2 / c f_{0} R_{i}
\end{array}\right)\right\}
$$

$i$ is the number of targets, $V_{e}$ and $V_{r i}$ are the amplitudes of the emitted and received signals, $\Delta F$ the sweep frequency, $F_{m}$ the modulation frequency, $f_{0}$ the carrier frequency, $c$ the light velocity, $k$ a mixer coefficient, $R_{i}$ and $V_{i}$ the ranges and radial velocities of the targets. The maximum range $R_{\max }$ before ambiguity is given by the period $T_{o b s}=1 / F_{m}$ of the modulation law:

$$
R_{\max }=c T_{o b s} / 2
$$

The radar is monostatic, i.e. a common antenna is used for both transmitting and receiving. It uses a horizontal scanning of the antenna to build panoramic images of the environment in PPI format. The rotation velocity of the antenna is $1 \mathrm{tr} / \mathrm{s}$, and 360 radar acquisitions per turn are achieved in order to build one panoramic image. The main characteristics of the PELICAN radar are provided in Table 1. A general view of the radar is presented in Fig. 1. Considering the weight and the size of the radar, it can be easily embarked aboard different kind of vehicles. The radar provides panoramic images are in PPI format. Raw radar images are corrected by the vehicle motion 
TABLE I. PELICAN RADAR CARACTERISTICS

\begin{tabular}{ll}
\hline Carrier frequency $f_{0}$ & $24.125 \mathrm{GHz}$ \\
\hline Transmitter power $P_{t}$ & $20 \mathrm{dBm}$ \\
\hline Frequency sweep $\Delta F$ & $250 \mathrm{MHz}$ \\
\hline Modulation frequency $F_{m}$ & $360 \mathrm{~Hz}$ \\
\hline Antenna gain $G$ & $20 \mathrm{~dB}$ \\
\hline Range & $3-100 \mathrm{~m}$ \\
\hline Half power beamwidth (horizontal / vertical) & $5^{\circ} / 25^{\circ}$ \\
\hline Distance resolution & $1 \mathrm{~m}$ \\
\hline Distance precision & $0.02 \mathrm{~m}$ \\
\hline Size (length-width-height), weight & $27-24-30 \mathrm{~cm}, 10 \mathrm{~kg}$ \\
\hline
\end{tabular}

thanks to a gyrometer and an odometer (if available). The speckle effect can also be reduced through the use of a multi look filter. An example of a panoramic radar image is presented in Fig. 2. In this image, the effect of the antenna aperture is clearly visible for largest distances.

The objectives of the R-SLAM mapping algorithm are to build a consistent map of the environment and to estimate the displacement of the radar within this map. The process is based on Simultaneous Localization And Mapping (SLAM) principles. Standard approaches to consider SLAM problems are typically based on Kalman or particle filters [8]. The objective of these methods is to compute in-line an estimation of the vehicle pose $x_{k}$ and an estimation of the map $M_{k}=\left[m_{0}\right.$, $\ldots, m_{k}$ ], considering the measurements $z_{k}$ observed at time $k$. These methods imply to recognize the same feature repeatedly during the process, thus it is necessary to detect persistent and reliable features within radar data. However, non-ambiguous target detection and identification based on radar signal interpretation are still open problems. Radar power/range spectra contain a large amount of information mixed with various sources of noise, which makes detection and tracking of particular landmarks not a simple problem. Moreover, in poorly structured environment, the clutter must be taken into account because it may be the only available information. In such situations, a detection approach based on threshold selection will fail. The main idea of the developed R-SLAM mapping algorithm is to use the high density of radar data through a scan-matching technique, in order to avoid the detection of particular landmarks [9]. The process is built with an incremental approach, including two main steps: (i) 3D correlation (map matching) between the current radar image and the global map in order to estimate the displacement (two translations and one rotation); (ii) merging process (map updating) in order to integrate the current radar image in the global map. An example of radar map construction is shown in Fig. 3.
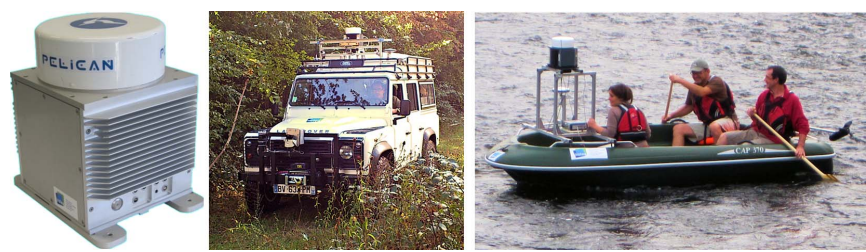

Fig. 1. General view of the PELICAN radar. Considering the weight and the size of the radar, it can be easily embarked aboard different kind of vehicles.

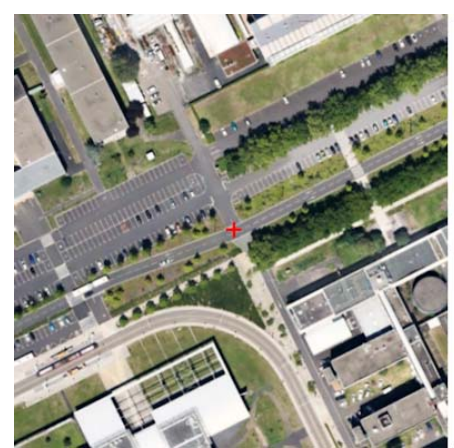

(a)

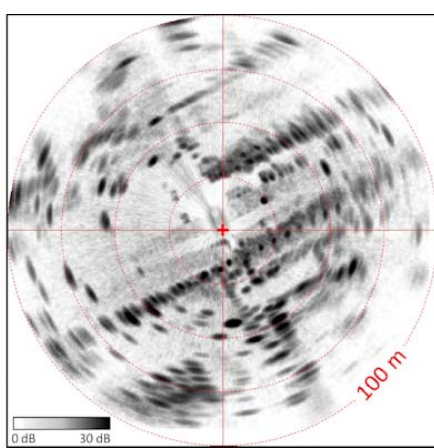

(b)
Fig. 2. Example of a panoramic radar image. (a) Aerial view of the test zone. The red cross indicates the radar's position. (b) Corresponding panoramic radar image. An anti-speckle filter has been applied.

\section{Distance RESOlUTION IMPROVEMENT}

With a classical FFT frequency analysis, the frequency resolution $\delta f$ is determined solely by the observation time $T_{o b s}$ :

$$
\delta f=1 / T_{o b s}, \text { with } T_{o b s}=N / f_{s}
$$

$N$ is the number of samples acquired and $f_{s}$ the sampling frequency. Considering a FMCW radar, the increase of $T_{o b s}$ (while maintaining the same sweep frequency $\Delta F$ ) leads to an increase of $\delta f$, but the distance resolution $\delta r$ remains the same because at the same time the maximum range is increased (see (2)).

With FMCW radars, the distance resolution $\delta r$ is solely determined by the sweep frequency $\Delta F[5]$ :

$$
\delta r=\mathrm{c} /(2 \Delta F)
$$

A simulation is presented in Fig. 4 using the model of signal described in (1), when considering two punctual targets located at ranges $39.75 \mathrm{~m}$ and $40.25 \mathrm{~m}$. With $\Delta F=200 \mathrm{MHz}$, the range resolution $\delta r$ is equal to $0.75 \mathrm{~m}$ and the FFT does not allow to separate the targets (Fig. 4(a)), even if $T_{o b s}$ is doubled (Fig. 4(b)). In Fig. 4(c), the multiplication by 2 of the sweep

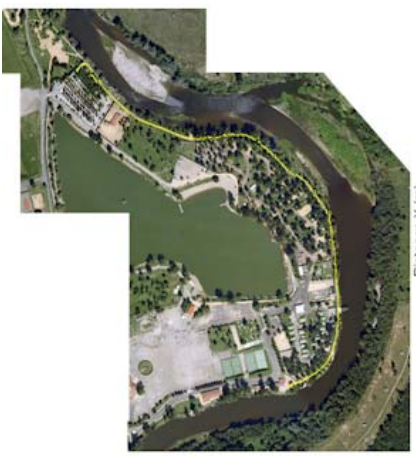

(a)

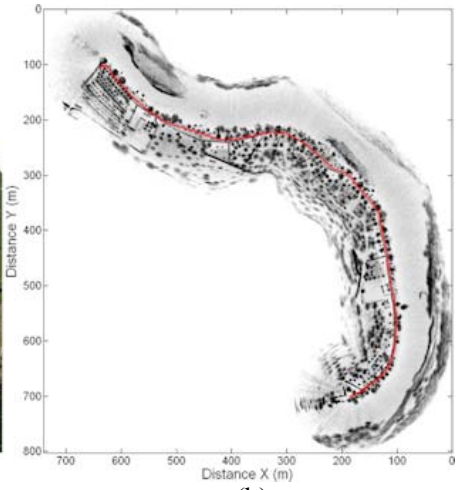

(b)
Fig. 3. Example of a radar map. (a) Aerial view of the test zone. The yellow dots cross indicate the GPS trajectory. (b) Computed radar map and trajectory (red dots). 


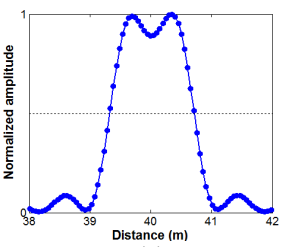

(a)

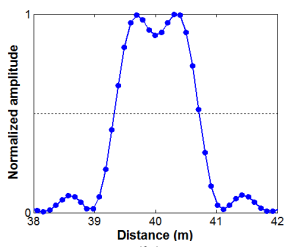

(b)

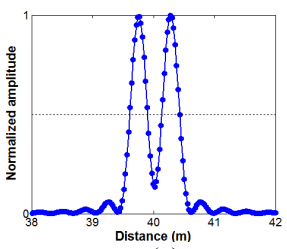

(c)
Fig. 4. Simulation of FFT radar spectra with 2 targets located at ranges $39.75 \mathrm{~m}$ and $40.25 \mathrm{~m}$. (a) $T_{o b s}=5.6 \mathrm{~ms}, \Delta F=200 \mathrm{MHz}$. (b) $T_{o b s}=11.2 \mathrm{~ms}, \Delta F=$ $200 \mathrm{MHz}$. (c) $\mathrm{T}_{\mathrm{obs}}=5.6 \mathrm{~ms}, \Delta F=400 \mathrm{MHz}$.

frequency $(\Delta F=400 \mathrm{MHz})$ allows to separate the targets.

It is therefore necessary to increase the sweep frequency to increase the distance resolution. But even if this increase is technologically possible, we have to deal with the French Telecomunications Regulatoty Autority (ARCEP). Within the $\mathrm{K}$-band, a free band of $250 \mathrm{MHz}(24.000-24.250 \mathrm{GHz})$ is available in France for civilian applications, completed with a limitation in transmitted power [10]. As the current value of bandwidth of the PELICAN radar is already $250 \mathrm{MHz}$, a second solution must be found to improve the distance resolution.

If the FFT is the basic tool for spectrum analysis due to its robustness and noise resistance, it exists different methods for high resolution spectrum estimation which overcome the limitations of FFT approach. For example, parametric methods such as MUSIC (Multiple Signal Classification) or ESPRIT (Estimation of Signal Parameter via Rotational Invariance Technique) take advantage of known parameters of the signal such as the number of spectral components [11,12].

ESPRIT is a spectrum estimation method based on the linear algebraic concept of subspaces. A subspace method uses the concept of eigenvector decomposition of the covariance matrix. The model of the signal is as a sum of sinusoids plus a white noise:

$$
\begin{aligned}
& \mathrm{x}=\sum_{i=1}^{p} A_{i} s_{i}+\eta \\
& \text { with } A_{i}=\left|A_{i}\right| \mathrm{e}^{j \phi_{i}} \text { and } s_{i}=\left[\begin{array}{llll}
1 & \mathrm{e}^{j \omega_{i}} & \ldots & \mathrm{e}^{j(N-1) \omega_{i}}
\end{array}\right]
\end{aligned}
$$

$A_{i}$ represents the magnitude and phase of the $i$-th frequency component, $p$ is the number of components, $N$ is the number of signal samples, $\eta$ the noise. The autocorrelation matrix of the signal is estimated from the signal samples with:

$$
R_{x}=\sum_{i=1}^{p} E\left\{A_{i} A_{i}^{*}\right\} s_{i} s_{i}^{T}+\sigma_{0}^{2} \mathrm{I}
$$

The $(N-p)$ smallest eigenvalues of the correlation matrix correspond to the noise subspace and the $p$ largest eigenvalues (all greater than $\sigma_{0}$, the noise variance) correspond to the signal subspace. The eigenvectors $\mathrm{E}$ of the autocorrelation matrix of the signal define two subspaces (signal and noise subspaces) by using two selector matrices $\Gamma_{1}$ and $\Gamma_{2}$ :

$$
\mathrm{S}_{1}=\Gamma_{1} \mathrm{E} \text { and } \mathrm{S}_{2}=\Gamma_{2} \mathrm{E}
$$

As the ESPRIT algorithm is based on naturally existing shift invariance between discrete time series, there is a rotational invariance between both subspaces which leads to the equation:

$$
\mathrm{S}_{1}=\Phi \mathrm{S}_{2}
$$

where

$$
\Phi=\left[\begin{array}{cccc}
\mathrm{e}^{j \omega_{1}} & 0 & \cdots & 0 \\
0 & \mathrm{e}^{j \omega_{2}} & \cdots & 0 \\
\vdots & \vdots & \ddots & \vdots \\
0 & 0 & & \mathrm{e}^{j \omega_{r}}
\end{array}\right]
$$

The matrix $\Phi$ contains all information about the $p$ components' frequencies.

A simulation based on signal model (1) is presented in Fig. 5. Three punctual targets are located at range $39.75 \mathrm{~m}$, 40.20m and 40.40m. The FFT spectrum analysis (see Fig. 5(a)) does not allow separating the targets, even if the sweep frequency $\Delta F$ is doubled (Fig. 5(b)). In Fig. 5(c), one can observe that the three targets are clearly localized when using the ESPRIT algorithm.

\section{ANGULAR RESOLUTION IMPROVEMENT}

Angular resolution is the minimum angular separation at which two equal targets can be separated when at the same range. The angular resolution characteristics are determined by the antenna beam width represented by the $-3 \mathrm{~dB}$ angle $\theta$ which is defined by the half-power $(-3 \mathrm{~dB})$ points. A rough estimation of the beam width is given by the ratio of the wavelength to the antenna size:

$$
\sin (\theta) \approx \lambda / d
$$

where $\lambda$ is the wavelength of the transmitted signal and $d$ the antenna's size. According to (10), we obtain a beam width of $4.1^{\circ}$ with the characteristics of the PELICAN antenna. It is a quite good approximation compared to the manufacturer's information of $5^{\circ}$. Considering (10), an increase of the angular resolution can be obtained with (i) a decrease of $\lambda$, or (ii) an increase of $d$. $\lambda$ and $d$ are parameters which cannot be modified due to external criteria (bandwidth limitation, maximum antenna's size, etc.), so another solution must be found. Synthetic Aperture Radar (SAR) method used with airborne or spaceborne radars is not applicable with the PELICAN radar

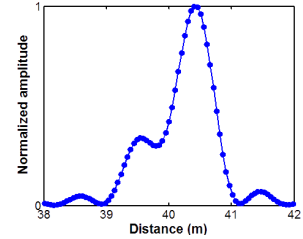

(a)

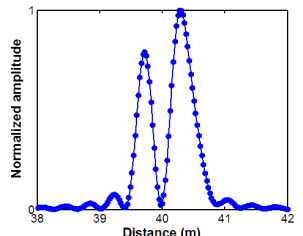

(b)

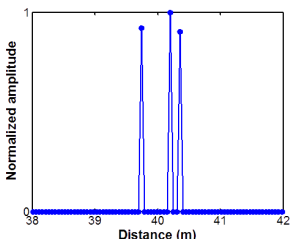

(c)
Fig. 5. Simulation of radar spectra with FFT and ESPRIT methods. Three punctual targets are located at ranges $39.75 \mathrm{~m}, 40.20 \mathrm{~m}$ and $40.40 \mathrm{~m}$.

$T_{o b s}=5.6 \mathrm{~ms}$. (a) FFT spectrum with $\Delta F=200 \mathrm{MHz}$. (b) FFT spectrum with $\Delta F=400 \mathrm{MHz}$. (c) ESPRIT spectrum with with $\Delta F=200 \mathrm{MHz}$. 
considering the rotation of the antenna and the behavior of the moving radar platform. As the panoramic radar image can be considered as the convolution between the antenna beam and the targets, a solution based on spatial deconvolution technique has been investigated.

The objective of these deconvolution techniques is to reverse the distortion that has been introduced by the imaging instrument (the antenna beam for the radar). This distortion can be expressed as

$$
g(x)=f(x) * h(x)+n(x)
$$

where $g$ is the acquired image, $f$ is the "real" image, $h$ is the point spread function (PSF) of the imaging system, and $n$ an additive noise. Several deconvolution algorithms are proposed in the literature to restore the image $f$. These algorithms can be non-iterative algorithms (regularized inverse-filtering algorithm [13], Wiener filtering algorithm [14], etc.), or iterative algorithms such as Richardson-Lucy algorithm $[15,16]$ or Carrington algorithm [17].

The Richardson-Lucy algorithm is a well-known Bayesianbased method for the deconvolution of images convolved with a known PSF. It is commonly used in astronomy domain (it is known to be used for the Hubble Space Telescope). Well adapted to the localization of point sources, it has also been used for the deconvolution of radar images [18]. The Richardson-Lucy algorithm follows the next procedure:

$$
f_{k+1}=f_{k} \cdot\left[g /\left(f_{k}^{*} h\right) \otimes h\right]
$$

where $f_{k+1}$ denotes the reconstructed image at the $k$-th iteration.

A simulation of deconvolution with Richardson-Lucy algorithm is presented in Fig. 6 . The $-3 \mathrm{~dB}$ antenna beam width is $5^{\circ}$, and a punctual target is located at distance $40 \mathrm{~m}$ from the radar. In Fig. 6(a), FFT spectra (computed during the rotation of the antenna) show the effect of the convolution between the punctual target and the antenna beam. The Richardson-Lucy algorithm (with 25 iterations) allows to recover the theoretical angular response of the punctual target (see Fig. 6(b)).

\section{RESUlTS}

The effect of distance and angular improvement based on high resolution spectra processing and Richardson-Lucy deconvolution, have been tested with data provided by the PELICAN radar. The overall process of the PELICAN radar has been modified in order to have the main following steps:

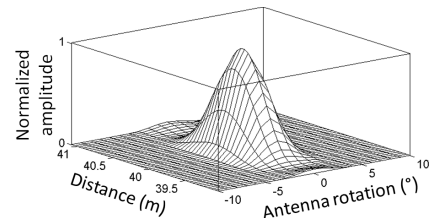

(a)

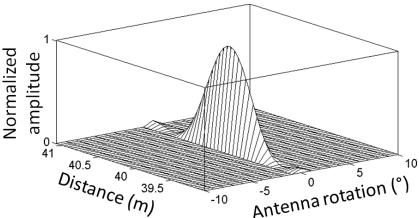

(b)
Fig. 6. Simulation of a Richardson-Lucy deconvolution considering a punctual target located at range $40.00 \mathrm{~m}$, and FFT spectral analysis. (a) Initial radar image. (b) Radar image with Richardson-Lucy deconvolution (25 iterations).
1) radar raw data acquisition
2) radar spectra estimation using ESPRIT algorithm
3) Richardson-Lucy deconvolution
4) R-SLAM algorithm

\section{A. PSF Construction}

The accuracy of the Richardson-Lucy deconvolution (and of any deconvolution algorithm) depends on the quality of used PSF. The PSF can be theoretically simulated from the antenna characteristics, or estimated from measurements of a punctual target.

The second approach has been selected. The PSF has been computed using the radar image from a Luneburg lens (see Fig. 8).

\section{B. Results Obtained with Canonical Targets}

The process has been firstly evaluated on a static panoramic radar image with two canonical targets (see Fig. 8). A Luneburg lens and a trihedral corner were positioned at $29 \mathrm{~m}$ from the radar. The targets are $2 \mathrm{~m}$ separated in the azimuth dimension, which corresponds to an azimuth angle of $4^{\circ}$.

Fig. 7(a) shows the initial FFT analysis, which points out the poor distance and angular resolutions. Both targets are not resolved. In Fig. 7(b), the Richardson-Lucy deconvolution is applied alone on the FFT spectra, and the improvement of the angular resolution is clearly visible. In Fig. 7(c), the FFT spectral analysis is replaced by the ESPRIT method. Fig. 7(d) is the combination of both signal processing methods: ESPRIT spectrum analysis followed by the Richardson-Lucy deconvolution. The punctual nature of the canonical targets is highlighted by this signal processing.

\section{Radar Maps Construction}

This method was tested on a dataset collected along a $2.5 \mathrm{~km}$ section of road between Romagnat and Opme (Auvergne, France). The PELICAN radar is positioned on the roof of an off-road vehicle (see Fig. 1). The mean velocity of the vehicle is $4 \mathrm{~m} / \mathrm{s}$. The panoramic radar images have been computed with two methods: FFT method on one hand, and \{ESPRIT + Richardson-Lucy deconvolution\} on the other hand. The computed image dataset has been used by the

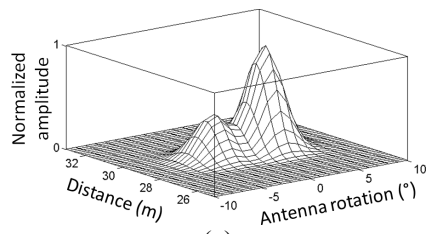

(a)

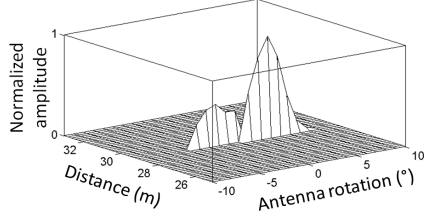

(c)

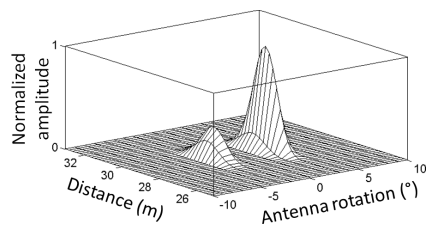

(b)

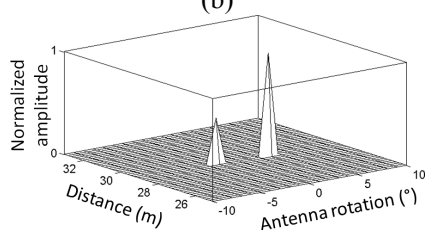

(d)
Fig. 7. Radar image obtained with two canonical targets at the same range and closely spaced in azimut. (a) FFT image. (b) Richardson-Lucy deconvolution. (c) ESPRIT spectral analysis. (d) ESPRIT + Richardson-Lucy deconvolution. 


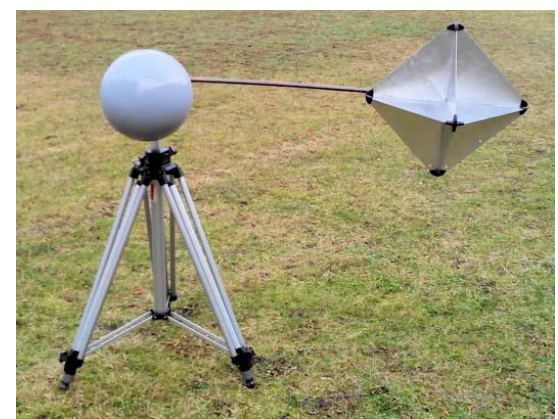

Fig. 8. Canonical reflectors used during the experiment: Luneburg lens (left) and trihedral corner (right).

R-SLAM algorithm to build the radar map of the covered zone. A part of the overall maps is presented in Fig. 9. The comparison between Fig. 9(b) and Fig. 9(c) highlights the improvement of the image resolution obtained with the \{ESPRIT + Richardson-Lucy deconvolution $\}$ method.

\section{CONCLUSION}

PELICAN is a real-aperture panoramic radar devoted to perception in outdoor environments. Its panoramic images are used by the R-SLAM algorithm in order to build consistent maps of the environment. The spatial resolution of the constructed maps is limited due to the distance and angular resolutions of the panoramic radar images.

To overcome the limitation in distance resolution introduced by the limited bandwidth of the transmitted signal, the high resolution ESPRIT parametric method has been used in place of the conventional FFT spectrum analysis. With a real aperture radar, the angular resolution is fixed by the size of the antenna beam width. As neither wavelength nor antenna size cannot be changed (due to external constraints), the angular resolution has been improved through the use of a Richardson-Lucy deconvolution. The simultaneous use of ESPRIT algorithm and Richardson-Lucy deconvolution allows to obtain radar maps with a higher spatial resolution.

\section{ACKNOWLEDGMENT}

This work is part of the "HypOp" reseach project, supported by the Laboratory of Excellence IMobS3 "Innovative Mobility: Smart and Sustainable Solutions".

\section{REFERENCES}

[1] T. Peynot, S. Scheding, and S. Terho, "The Marulan Data Sets: MultiSensor Perception in Natural Environment with challenging conditions," The International Journal of Robotics Research, vol. 29(13), pp. 1602$1607,2010$.

[2] G. Reina, J. Underwood, G. Brooker, and H. Durrant-Whyte, "Radarbased perception for autonomous outdoor vehicles," Journal of Field Robotics, vol. 28(6), pp. 894-913, 2011.

[3] D. Vivet, P. Checchin, R. Chapuis, P. Faure, R. Rouveure, and M.O. Monod,"A mobile ground-based radar sensor for detection and tracking of moving objects," EURASIP Journal on Advances in Signal Processing, vol. 45, 13 p., 2012.

[4] M. Jaud, R. Rouveure, P. Faure, and M.O. Monod, "Methods for FMCW radar map georeferencing," ISPRS Journal of Photogrammetry and Remote Sensing, vol. 84, pp. 33-42, 2013.

[5] M. I. Skolnik, "Introduction to radar systems," In Electrical Engineering Series, McGraw-Hill International Editions, 1980.

[6] J G. Reina, J. Underwood, G. Brooker, and H. Durrant-Whyte, "RadarBased Perception for Autonomous Outdoor Vehicles," Journal of Field Robotics, vol. 28(6), pp. 894-913, 2011.

[7] M.O. Monod, P. Faure, and R. Rouveure, "Intertwined Linear Frequency Modulated Radar and Simulator for Outdoor Robotics Applications," IEEE International Radar Conference (RADAR'09), 6 p., 2009.

[8] H. Durrant-Whyte, and T. Bailey, "Simultaneous Localization and Mapping (SLAM): Part I - The Essential Algorithms," Robotics and Automation Magazine, vol. 13(2), pp. 99-110, 2006.

[9] R. Rouveure, M.O. Monod, and P. Faure, "High Resolution Mapping of the Environment with a Ground-Based Radar Imager," IEEE International Radar Conference (RADAR'09), 6 p., 2009.

[10] Agence Nationale des Fréquences, "TABLEAU NATIONAL DE REPARTITION DES BANDES DE FREQUENCES,” Edition 2013.

[11] R.O. Schmidt, "Multiple Emitter Location and Signal Parameter Estimation," IEEE Transactions on Antennas and Propagation, vol. 34(3), pp. 276-280, 1986.

[12] S. Shahbazpanahi, S. Valaee, and M. H. Bastani, "Distributed Source Localization Using ESPRIT Algorithm," IEEE Transactions on Signal Processing, vol. 49(10), pp. 2169,2178, 2001.

[13] C. Preza, M. Miller, I. Thomas, and J. McNally, "Regularized linear method for reconstruction of three-dimensional microscopic objects from optical sections,” J. Opt. Soc. Am. A., vol. 9, pp. 219-228, 1992.

[14] P.J. Shaw, and D.J. Rawlins, "The point-spread function of a confocal microscope: its measurement and use in deconvolution of 3-D data," J. Microsc., vol. 163(2), pp. 151-165, 1991

[15] W.H. Richardson, "Bayesian-based iterative method of image restoration," Journal of the Optical Society of America, vol. 62(1), pp. 55-59, 1972.

[16] L.B. Lucy, "An iterative technique for the rectification of observed distributions," The Astronomical Journal, vol. 79(6), pp. 745-754, 1974.

[17] W. Carrington, R. Lynch, E. Moore, G. Isenberg, K. Fogarty, and F. Fay, "Superresolution three-dimensional images of fluorescence in cells with minimal light exposure," Science, vol. 268, pp. 1483-1487, 1995.

[18] F. Diewald, J. Klappstein, J. Dickmann, and K. Dietmayer, “An Adaption of the Lucy-Richardson Deconvolution Algorithm to Noncentral Chi-Square Distributed Data," IAPR Conference on Machine Vision Applications, pp. 389-392, 2011. 
(a)

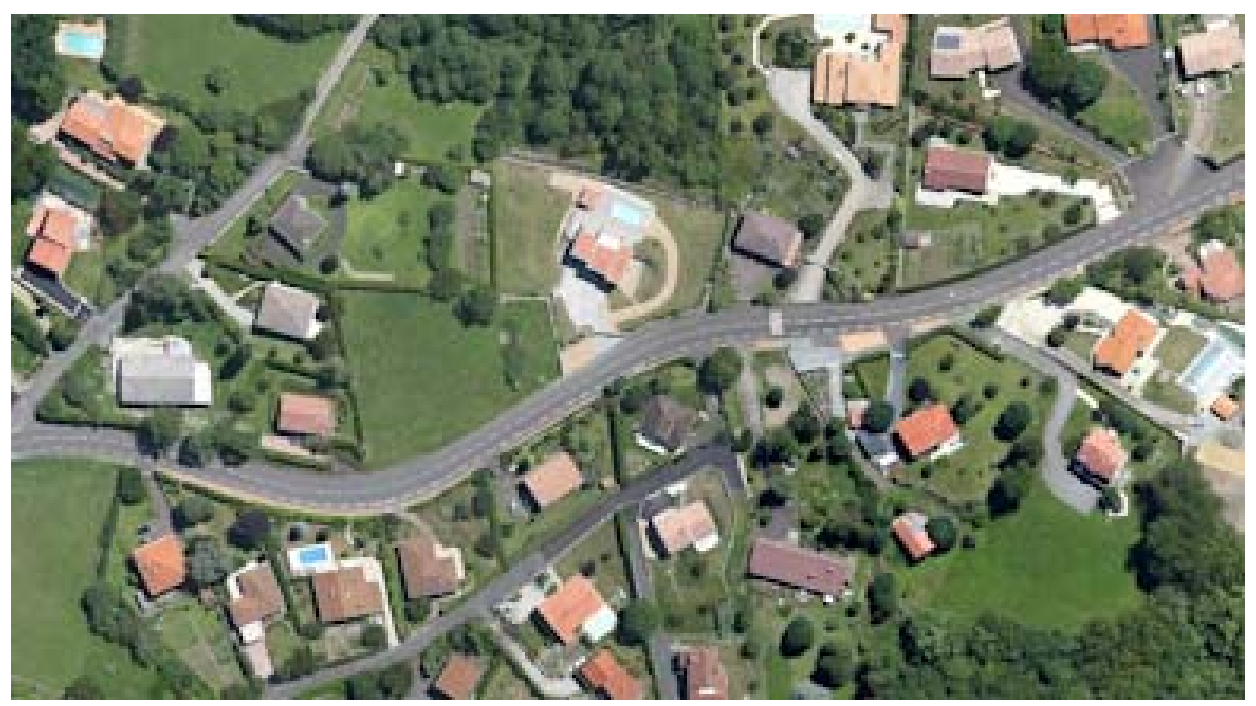

(b)
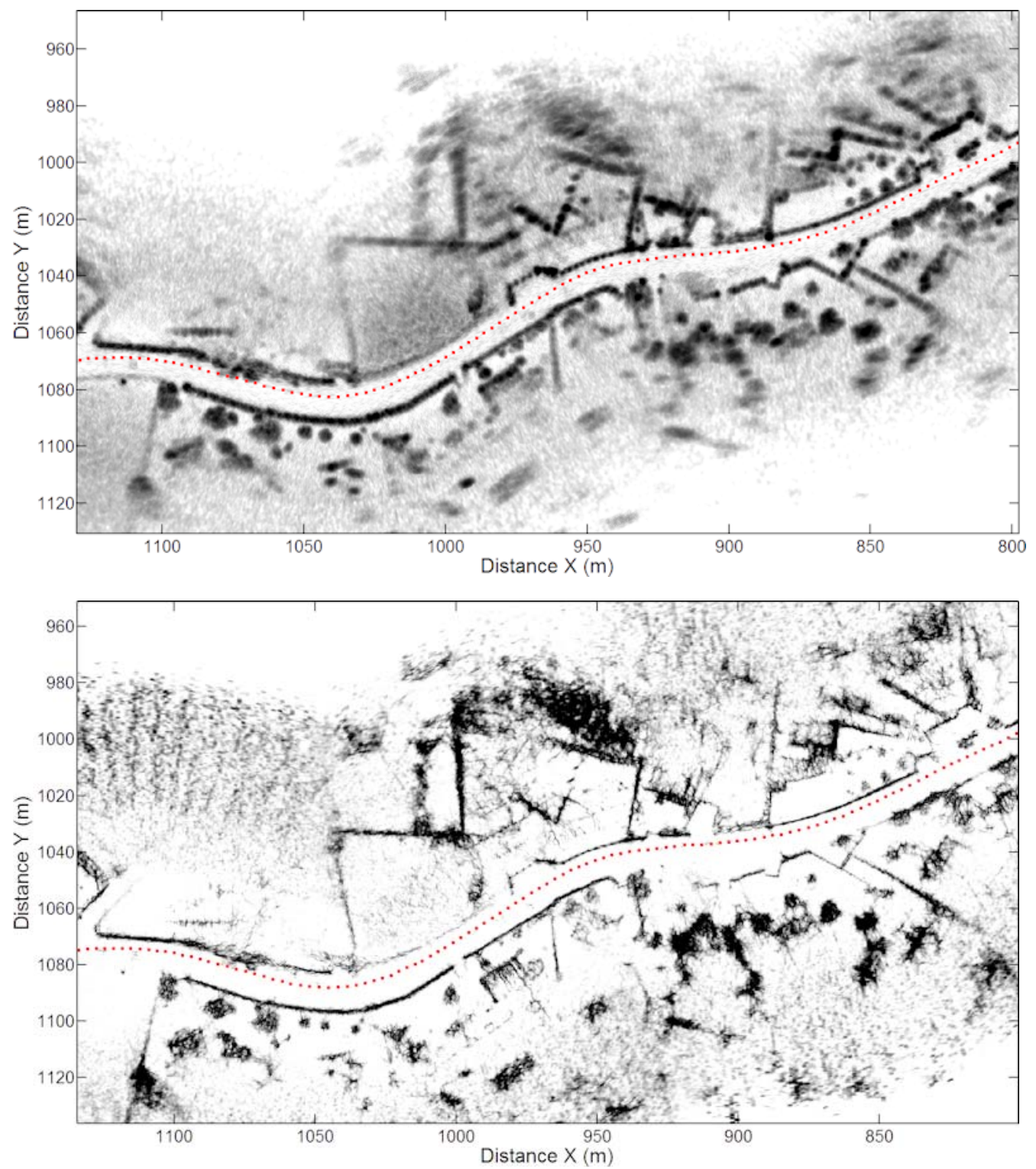

Fig. 9. Examples of radar maps obtained with R-SLAM algorithm. (a) Aerial view of the zone (Goggle Earth). (b) Radar map and trajectory (red dots) obtained with a FFT spectral analysis. (c) Radar map and trajectory (red dots) obtained when using ESPRIT and Lucy-Richardson signal processing techniques. 